\title{
Drug-Induced Methemoglobinemia: a Case Report and Review of Literature
}

\author{
Ahmed Zedan MD, Sabry Omar MD, Mahmoud Fenire MD
}

\begin{abstract}
Drugs, including those used during diagnostic procedures, can have adverse effects and potentially serious side-effects, especially in complicated patients with significant comorbidity. Benzocaine is frequently used as an oropharyngeal anesthetic agent during bronchoscopy, transesophageal echocardiography, and upper GI endoscopy and can cause methemoglobinemia, a potentially life-threatening event if not diagnosed and treated quickly. Co-oximetry is the gold standard for the diagnosis of methemoglobinemia and can quantitate blood levels, which in turn correlate with the clinical presentation and the urgency for treatment. Methylene blue is the treatment of choice for methemoglobinemia. In this case report we discuss the pathophysiology, the clinical presentation, the diagnosis, and the treatment of benzocaine-induced methemoglobinemia.
\end{abstract}

Key words: methemoglobinemia, methylene blue, tissue hypoxia, benzocaine

\section{INTRODUCTION}

Methemoglobinemia (hemoglobin with oxidized iron, $\mathrm{Fe}^{+++}$) is a rare cause of central cyanosis that can be life threatening. Under normal physiologic conditions, methemoglobin has a half-life of 55 minutes, and levels are below $2 \%$. ${ }^{1,2}$ Methemoglobinemia can be either acquired or congenital. Acquired methemoglobinemia occurs when the rate of methemoglobin formation exceeds the rate of its reduction and is often caused by pharmacologic agents. Dapsone is used to treat infectious diseases such as pneumocystis and some autoimmune disorders and can cause methemoglobinemia. The topical anesthetic benzocaine is commonly used in some procedures, like bronchoscopy or transesophageal echocardiogram, and can also cause methemoglobinemia. Therefore, all health care providers, especially those participating in these procedures, should know the association between routine procedures and methemoglobinemia for early recognition and treatment. In this report we discuss a case of acquired drug-induced methemoglobinemia associated with the use of benzocaine for transesophageal echocardiography (TEE).

\section{Case Presenttion}

A 42-year-old man with a past medical history of type 2 diabetes mellitus, hypertension, drug abuse, and left below-knee amputation presented to the emergency department with his right leg "dragging" and altered speech that started a few hours before his arrival. An immediate CT scan of the brain didn't show any evidence of intracranial pathology. A MRI showed an acute nonhemorrhagic left anterior cerebral artery infarct and acute nonhemorrhagic bilateral occipital infarcts. On physical examination his temperature was $98.5^{\circ} \mathrm{F}$, blood pressure $173 / 79 \mathrm{mmHg}$, pulse rate 73 beats per minute, and respiratory rate 16 breaths per 
minute. His heart and lung examinations were within normal limits, and his oxygen saturation was $97 \%$ on room air. Laboratory finding showed WBC $7.5 \mathrm{k} / \mu \mathrm{L}$, hemoglobin $13.8 \mathrm{gm} / \mathrm{dl}$, normal troponin, and brain natriuretic peptide $750 \mathrm{pg} / \mathrm{ml}(\mathrm{nl}<124 \mathrm{pg} / \mathrm{ml})$. His 12lead electrocardiogram and chest $\mathrm{x}$-ray were within normal limits. The patient was admitted for treatment and started on simvastatin, aspirin, Procardia XL, hydrochlorothiazide, and enoxaparin. His work-up for an acute stroke included transesophageal echocardiography. Before this procedure, his vital signs were: temperature $98{ }^{\circ} \mathrm{F}$, blood pressure $145 / 75 \mathrm{mmHg}$, pulse 78 beats per minute, and respiratory rate 15 breaths per minute. During the procedure benzocaine $20 \%$ spray was used for oropharyngeal anesthesia. Oxygen saturations by pulse oximetry before, after, and during the procedure were $97 \%$ on $2 \mathrm{~L}$ oxygen nasal cannula. The echocardiogram did not show any evidence of cardiac structural abnormalities with a normal ejection fraction (60-64\%). An hour after the procedure the patient developed central cyanosis; his oxygen saturation was $85 \%$ by pulse oximetry. His blood pressure was 152/77 mmHg, heart rate 105 beats per minute, and respiratory rate 32 breaths per minute. His cardiovascular and chest examination was within normal limits. The patient felt uncomfortable and lethargic. Oxygen was administered with a non-rebreathing face mask initially at $10 \mathrm{~L} /$ minute and then at $15 \mathrm{~L} /$ minute when the cyanosis did not resolve. A chest x-ray was normal, an electrocardiogram showed sinus tachycardia, and a chest CT with PE protocol showed no evidence of pulmonary emboli. ABG results included $\mathrm{pH} 7.45, \mathrm{PCO}_{2} 42 \mathrm{mmHg}$, and $\mathrm{PO}_{2} 282 \mathrm{mmHg}$. Troponin, CK, and CKMB levels were within normal limits. Methemoglobinemia was suspected; co-oximetry revealed a methemoglobin level of $37 \%$ (normal $0.6-2.5 \%$ ). Methylene blue was started, and after eight hours his methemoglobin level decreased to $6.6 \%$. The patient's oxygen requirements decreased to $3 \mathrm{~L} /$ minute by nasal cannula. Within 12 hours, his cyanosis resolved. A repeat arterial blood gas the next morning demonstrated a methemoglobin level of $1.1 \%$ by co-oximetry.

\section{Discussion}

Hemoglobin is a tetramer composed of alpha, beta, gamma, or delta chains. The most common form of hemoglobin in adults $(\mathrm{HbA})$ consists of two $\alpha$ and two $\beta$ chains. Each hemoglobin chain is formed by a globin polypeptide linked to a heme group, which is formed by a complex of a protoporphyrin IX ring and one atom of ferrous iron $\left(\mathrm{Fe}^{+2}\right)$. Thus, each $\mathrm{Hb}$ molecule has four atoms of iron. Each ferrous iron can reversibly link one $\mathrm{O}_{2}$ molecule, and four molecules of $\mathrm{O}_{2}$ are transported by each hemoglobin molecule.

Hemoglobin is constantly being oxidized, but intrinsic reducing systems maintain the levels of methemoglobin under $2 \%$. NADH-methemoglobin reductase (NADH-NR), a system with two enzymes- cytochrome B5 and cytochrome B5-reductase, maintains endogenous reduction of methemoglobinemia and accounts for $99 \%$ of the reducing activity. NADH-methemoglobin reductase transfers one electron from $\mathrm{NADH}$ to methemoglobin, changing it into reduced hemoglobin. Other systems also help maintain low levels of methemoglobin, and these include ascorbic acid, glutathione, and NADPH dehydrogenase. Under normal conditions these pathways are less significant but become important when NADH-MR is disrupted. When $\mathrm{Fe}^{+2}$ is oxidized to ferric iron $\left(\mathrm{Fe}^{+3}\right)$, it cannot bind oxygen. Ferric iron also causes an allosteric change in the heme portion of partially oxidized hemoglobin and increases $\mathrm{O}_{2}$ affinity. This methemoglobin shifts the dissociation curve of partially oxidized hemoglobin to the left and reduces the release of $\mathrm{O}_{2}$ in the tissues leading to tissue hypoxia.

Hereditary methemoglobinemia is rare. The most common cause of congenital methemoglobinemia is cytochrome b5 reductase deficiency (type Ib5R) which is endemic in certain Native American tribes. Most cases of methemoglobinemia are acquired and occur after exposure to drugs or toxins. Topical benzocaine, an oropharyngeal anesthetic medication, is probably the most common cause of acquired methemoglobinemia. A study of 886 patients undergoing TEE reported that methemoglobinelevation occurred in four patients $(0.115 \%))^{3}$ Ash-Bernal 
reviewed 138 cases of acquired methemoglobinemia; 56 cases were taking dapsone for the prevention or treatment of an infection caused by Pneumocystis jiroveci. The next group comprised patients undergoing surgical procedures and diagnostic investigations, such as cardiac catheterization. The highest methemoglobin concentrations were observed in five patients in whom a $20 \%$ benzocaine spray had been used. One patient subsequently died, and three others required long-term treatment. ${ }^{4}$ At the Mayo Clinic during a six and one-half year period, 28,478 patients underwent TEEs; 19 cases had methemoglobinemia with a mean methemoglobin level of $32 \% \pm 15 \%$. Eighteen patients received methylene blue $(1.3 \pm 0.4$ $\mathrm{mg} / \mathrm{kg}$ of body weight) with resolution of symptoms and signs. One patient resolved spontaneously. ${ }^{5}$

Clinical manifestations of methemoglobinemia reflect the reduction in $\mathrm{O}_{2}$-carrying capacity and associated tissue hypoxia. The presentation of methemoglobinemia depends on both the methemoglobin concentrations and patient co-morbidity. Methemoglobin concentrations under $15 \%$ cause only a grayish pigmentation of the skin. Above $12 \%$ to $15 \%$, the blood is a brown chocolate color, and patients have central cyanosis unresponsive to the administration of $\mathrm{O}_{2}$. With methemoglobin levels above $20 \%$ to $30 \%$ neurological (dizziness, headache, anxiety, somnolence, and seizures) and cardiovascular symptoms (dyspnea, low cardiac output symptoms) start to appear. As levels of methemoglobin increase, the patient may have a reduced level of consciousness, respiratory depression, shock, and death. Levels of methemoglobin above $70 \%$ are usually fatal. Anemia makes patients more sensitive to methemoglobinemia by reducing the functional stores of hemoglobin.

Table: Clinical presentation of methemoglobinemia based on methemoglobin level in blood. ${ }^{6}$

\begin{tabular}{|l|l|}
\hline \multicolumn{1}{|c|}{ Methemoglobin \% } & \multicolumn{1}{|c|}{ Symptoms } \\
\hline$<\mathbf{1 5 \%}$ & Asymptomatic \\
\hline $\mathbf{2 0}-\mathbf{3 0} \%$ & Cyanosis, confusion, syncope, dizziness. \\
\hline $\mathbf{3 0}-\mathbf{5 0} \%$ & Shortness of breath and headache. \\
\hline $\mathbf{5 0 - 7 0 \%}$ & Dysrhythmias, seizure, stupor, coma \\
\hline Over $\mathbf{7 0} \%$ & Death \\
\hline
\end{tabular}

Corresponding author: Ahmed Zedan MD Contact Information: ahmed.zedan@ttuhsc.edu DOI: 10.12746/swrccc2014.0205.060
Methemoglobinemia should be suspected in patients with central cyanosis, tachypnea, and low $\mathrm{O}_{2}$ saturations on pulse oximetry, especially if not responding to $100 \%$ oxygen. Other common causes like cardiopulmonary disorders also need to be considered. Arterial blood gases with co-oximetry are the gold standard for the diagnosis of methemoglobinemia and measure the concentration of different types of hemoglobin in blood through spectrophotometry using different wavelengths. This technology is based on the Lambert-Beer law that correlates 
the concentration of a dissolved substance with the intensity of the light transmitted through a solution. Other ways to confirm the diagnosis include apositive Kronenberg test result or confirmation of the oxygen saturation gap. ${ }^{6}$

The treatment of methemoglobinemia should be guided by the severity of the disorder. Blood levels of methemoglobin represent a secondary parameter in the decision about treatment. Usually, the symptoms are mild. In those cases, treatment consists of removing the inducing agent, administration of high-flow $\mathrm{O}_{2}$, observation, and periodic co-oximetry assessment. ${ }^{7}$ After discontinuation of the causative agent, the methemoglobin level usually returns to baseline levels within 36 hours. The use of supplementary $\mathrm{O}_{2}$ increases plasma levels of dissolved $\mathrm{O}_{2}$ and increases diffusion and oxygen delivery. With significant clinical manifestations (e.g., dizziness, headache, anxiety, dyspnea, symptoms of low cardiac output, somnolence, and seizures), methylene blue should be started as an antidote. Some authors suggest that methylene blue should be used with methemoglobin level above $30 \%$ regardless of symptoms. This is especially recommended when the patient is unconscious (e.g., head trauma, deep sedation, or general anesthesia). Methylene blue is given intravenously in a dose of 1 to $2 \mathrm{mg} / \mathrm{kg}$ over five minutes. During its use, the alternative enzymatic system (NADPH methemoglobin reductase) becomes fundamental in the reduction of methemoglobin. Methylene blue activates NADPH methemoglobin reductase which reduces methylene blue to methylene leucoblue, which transforms methemoglobin to reduced hemoglobin by a non-enzymatic mechanism. ${ }^{8,9}$ Additional doses can be administered every hour, if necessary, up to a maximum total dose of $7 \mathrm{mg} / \mathrm{kg}$. If the dose exceeds $15 \mathrm{mg} / \mathrm{kg}$, it can cause direct damage of red blood cells and hemolysis with Heinz body formation.

Methylene blue should not be administered to patients with known glucose 6-phosphate dehydrogenase (G6PD) deficiency, because methylene blue depends upon NADPH generated by G6PD in the reduction process of methemoglobin. As a re- sult, this medication may not only be ineffective but is also potentially dangerous, since methylene blue has an oxidant potential that may induce hemolysis in G6PD deficient subjects. ${ }^{10}$ The levels of methemoglobin should fall significantly 30 to 60 minutes after the first dose. This drug should be administered carefully in patients with renal failure, since both methylene blue and leucoblue are slowly excreted by the kidneys. During treatment, the urine has a bluish tint. The same occurs, in varying degrees, to the skin and mucous membranes, hindering the interpretation of cyanosis after the treatment. Other treatments for methemoglobinemia include ascorbic acid with the intravenous administration of 300 to $1,000 \mathrm{mg}$ daily in selected cases of non-severe NADH-MR deficiency. Blood transfusion or exchange transfusion may be helpful in patients who are in shock. Hyperbaric oxygen has been used with anecdotal success in severe cases. $^{11}$

\section{Conclusion}

Methemoglobinemia is an uncommon cause of central cyanosis that can be a life threatening condition if not considered in the differential diagnosis and work-up of patients who are refractory to oxygen administration. Benzocaine, a commonly used atopical anesthetic, and dapsone can cause methemoglobinemia as rare life threatening side effect. Methylene blue should be available in all areas where benzocaine is used to allow rapid and prompt management of this condition.

\section{KEYPOINTS}

1. Acquired methemoglobinemia can reduce $\mathrm{O}_{2}$ delivery to tissues.

2. Benzocaine and dapsone are frequent causes of acquired methemoglobinemia.

3. These patients develop central cyanosis of body and multiple neurological symptoms. Coma, seizures, and death can occur.

4. Methylene blue is the treatment of choice. 
Author Affiliation: Ahmed Zedan, Sabry Omar and Mahmoud Fenire are internal medicine residents at TTUHSC in Lubbock, TX.

Received: $12 / 01 / 2013$

Accepted: 01/01/2014

Reviewers: Victor Test MD, Kenneth Nugent MD

Published electronically: 01/15/2014

Conflict of Interest Disclosures: None
11. Goldstein GM, Doull J. Treatment of nitrite-induced methemoglobinemia with hyperbaric oxygen. Proc Soc Exp Biol Med 1971; 138:137

\section{REFERENCES}

1. Ellenhorn MJ. Ellenhorn's Medical Toxicology: Diagnosis and Treatment of Human Poisoning. 2nd ed. Baltimore: Williams \& Wilkins; 1997. p. 1496-9

2. Coleman MD, Coleman NA. Drug induced methemoglobinemia. Treatment issues. Drug Saf 1996; 14:394-405

3. Vallurupalli S, Das S., Manchanda S. Infection and the risk of Topical anesthetic induced clinically significant methemoglobinemia after transesophageal echocardiography. Echocardiography 2009; 31. [Epub ahead of print].

4. Ash-Bernal R., Wise R., Wright S.M. Acquired methemoglobinemia a retrospective series of 138 cases at 2 teaching hospitals. Medicine 2004; 83: 265-273

5. Kane GC, Hoehn SM, Behrenbeck TR, Mulvagh SL. Arch Intern Med. 2007 Oct 8; 167(18):1977-82

6. Adams V, Marley J, McCarroll C. Prilocaine induced methaemoglobinaemia in a medically compromised patient. Was this an inevitable consequence of the dose administered? Br Dent $J$ 2007; 203(10): 585-7

7. Khan NA, Kruse JA. Methemoglobinemia induced by topical anesthesia: a case report and review. Am J Med Sci 1999; 318:415-8

8. Agarwal N, Nagel RL, Prchal JT. Dyshemoglobinemias. In: Disorders of Hemoglobin: Genetics, Pathophysiology, and Clinical Management, 2nd ed, Steinberg M (Ed), 2009. p.607

9. Kane GC, Hoehn SM, Behrenbeck TR, Mulvagh SL. Benzocaine-induced methemoglobinemia based on the Mayo Clinic experience from 28478 transesophageal echocardiograms: incidence, outcomes, and predisposing factors. Arch Intern Med 2007; 167:1977

10. Rosen PJ, Johnson C, McGehee WG, Beutler E. Failure of methylene blue treatment in toxic methemoglobinemia. Association with glucose-6-phosphate dehydrogenase deficiency. Ann Intern Med 1971; 75:83 